\title{
Prosoziale Circuits im Gehirn als therapeutische Targets - Oxytocin als Substanz der Zukunft?
}

Oxytocin ist ein körpereigenes Nona-Peptidhormon, das speziesübergreifend regulierende Einflüsse auf zahlreiche Körperfunktionen und soziale Verhaltensweisen entfaltet. Oxytocin (OT) wird in magnozelIulären Neuronen der paraventrikulären und supraoptischen Nuklei des Hypothalamus synthetisiert, axonal zur posterioren Hypophyse transportiert und von dort aus in den Blutkreislauf freigesetzt. OT-Rezeptoren finden sich im Tiermodell in zwei für das Sozialverhalten wichtigen Netzwerken: 1. Mediale Amygdala, bed nucleus of the stria terminalis (BNST), Hypothalamus etc. und damit in einem Netzwerk, das u.a. Elternschaft, sexuelles und aggressives Verhalten reguliert und 2. basolaterale Amygdala, Insula, BNST, ventrales Striatum, präfrontaler Cortex etc. und damit in einem Netzwerk, das in die Regulation der Salienz von eingehenden Stimuli involviert ist. Entsprechend der heute vielfach vertretenen Salienz-Hypothese befrachtet OT eintreffende sensorische Reize entsprechend ihrer Relevanz für das Individuum mit attentionalen Ressourcen. OT entfaltet nicht nur eine Wirkung in der Verarbeitung sozialer Reize, sondern auch solchen, die direkt verhaltensrelevant in Bezug auf Annäherung und Vermeidung (z.B. Schmerzreize) sind.

Die Dichte an OT-Rezeptoren, so legen es Studien an Säugetieren nahe, hängt von elterlicher Zuwendung und frühkindlichen Bindungserfahrungen $a b$; die Expression von OT-Rezeptoren könnte damit Einfluss auf die Ausbildung von spezifischen, das Sozialverhalten bestimmenden Persönlichkeitsmerkmalen nehmen. So erstaunt es nicht, dass die Rezeptorexpression interindividual sehr variabel ist und damit eine wichtige Rolle in der Ausbildung der Mannigfaltigkeit sozialen Verhaltens beim Menschen spielen könnte.

In funktionellen Bildgebungsuntersuchungen konnten OT-Effekte v.a. in der Amygdala, aber auch in der Insula als zentrale Strukturen des Salienznetzwerkes identifiziert werden. So konnte in sozialen Bedrohungsparadigmen eine Modulation der cortico-amygdalaren neuronalen Aktivität einschließlich ihrer Konnektivität mit Auswirkungen auf soziales Vermeidungsverhalten nachgewiesen werden. Entsprechend wurden OT-Effekte bei Patienten mit sozialer Phobie untersucht. Welche Richtung die Effekte allerdings nehmen, hängt interessanterweise von den individuellen Verhaltensdispositionen in Verbindung mit evolutionär sinnvollen Verhaltenszielen ab; so zeigen Patienten mit erhöhter reaktiver Aggressivität stärkeres Vermeidungsverhalten nach OT-Gabe, während Individuen mit Autismus-Spektrumstörungen sich sozialen Reizen leichter annähern können. Auch wirkt OT auf das mesokorticolimbische Netzwerk, das soziales Annäherungsverhalten in seiner Belohnungspotenz moduliert.

Eine besonders umfangreiche Datenbasis liegt zu OT-Effekten auf die Gesichtsverarbeitung vor. Mimische Emotionen sind im sozialen Zusammenleben sehr bedeutsame Signale, die emotionale Zustände, aber auch Intentionen Anderer und potenzielle Gefahr ausdrücken. Die heutige Datenlage verweist darauf, dass OT die Erkennung mimischer Emotionen vor allem durch eine Modulierung der Verarbeitung der Augenregion beeinflusst, welche für den Ausdruck von Angst und Ärger, und damit Emotionen, die im Kontext von sozialer Bedrohung bedeutsam sind, besonders relevant ist. Entsprechend konnte auf der Verhaltensebene eine Beeinflussung der Anzahl und Dauer von Blickbewegungen auf die Augenregion nachgewiesen werden, die auf neuronaler Ebene mit einer Modulation der Amygdala-Aktivität einhergeht. Bedenkt man die Bedeutung, die Angst- und Ärger-Emotionen in der Entstehung und Beherrschung von aggressiven Impulsen haben, so legt dies die Prüfung einer therapeutischen Potenz bei Störungen nahe, die mit reaktiver Aggressivität einhergehen. Nicht zu unterschätzen ist allerdings die enge Verflechtung von OT-Effekten mit Kontextfaktoren, wie z.B. Aggressivität innerhalb oder außerhalb der eigenen sozialen Gruppe.
Zusammenfassend sprechen viele Befunde dafür, dass OT sozial-kognitive Funktionen unterschiedlicher Komplexität, von der Gesichtsverarbeitung bis hin zu Empathie und kooperativem Verhalten beeinflusst, und zwar im Sinne der Optimierung adaptiven Verhaltens in der sozialen Umwelt in Übereinstimmung mit evolutionär verwurzelten Motiven.

Bevor allerdings die Anwendung beim Menschen auf einen breiten Weg gebracht werden kann, müssen manche methodischen Probleme gelöst werden. Zum einen müsste der genaue Transport- bzw. Wirkungsweg von intranasal verabreichtem OT hin zu den entscheidenden Hirnnetzwerken aufgeklärt werden. Zum anderen beruhen Annahmen über die Verteilung von OT-Rezeptoren auf wenigen kleinen post mortem-Studien, die Befunde an $\mathrm{Na}$ gern weitgehend am Menschen replizieren konnten. Schließlich ist die Pharmakodynamik weitgehend unklar, da nur einzelne Studien parallel OT-Spiegel in Blut, Speichel und Liquor miteinander verbunden haben. Innovative Bildgebungsmethoden wie das „arterial spin labeling magnetic resonance imaging (ASL-MR)“, das sich eines intrinsischen Tracers bedient, sind auf dem Weg und konnten OT-induzierte Veränderungen in zerebralen Netzwerken, die für Salienz und affektive Empathie bedeutsam sind, quantifizieren. Zudem ist das Zusammenspiel zwischen OT und dopaminergen, serotonergen und glutamatergen Transmittersystemen aufzudecken, die für die dargestellten Effekte auf das Sozialverhalten entscheidend sein könnten. Schließlich sind die engen Interaktionen mit den Geschlechtshormonen zu spezifizieren und könnten erklären, warum sich OT-Wirkungen auf soziale Funktionen bei Männern und Frauen in Studien z. T. geradezu konträr darstellen.

\section{Interessenkonflikte}

Es bestehen keine Interessenkonflikte. 
Autor

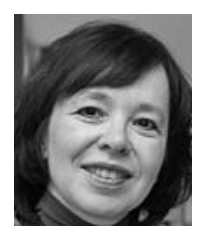

Sabine C. Herpertz

Lehrstuhl für Psychiatrie und

ärztliche Direktion an der Klinik

für Allgemeine Psychiatrie,

Universitätsklinikum Heidelberg
Korrespondenzadresse

Prof. Dr. Sabine C. Herpertz

Lehrstuhl für Psychiatrie und

ärztliche Direktion

Klinik für Allgemeine Psychiatrie

Universitätsklinikum Heidelberg

Voßstraße 2

69115 Heidelberg

sabine.herpertz@uni-heidelberg.de 